\title{
Perceived Stress and Psychological Impact Among Healthcare Workers at a Tertiaty Hospital in China During the COVID-19 Outbreak: The Moderating Role of Resilience and Social Support
}

\author{
Qiaoyang Zhang, Guanzhong Dong, Weifen Meng, Zhuoyou Chen, Yin Cao ${ }^{\star \dagger}$ and \\ Min Zhang *t
}

The Affiliated Changzhou No. 2 People's Hospital of Nanjing Medical University, Changzhou, China

\section{OPEN ACCESS}

Edited by:

Jutta Lindert,

University of Applied Sciences Emden

Leer, Germany

Reviewed by:

Cai-Lan Hou,

Guangdong Mental Health

Center, China

Muhammed Elhadi,

University of Tripoli, Libya

${ }^{*}$ Correspondence:

Yin Cao

czcaoyin@163.com

Min Zhang

zhangmin0411@njmu.edu.cn

these authors have contributed equally to this work

Specialty section: This article was submitted to Public Mental Health,

a section of the journal

Frontiers in Psychiatry

Received: 09 June 2020 Accepted: 29 December 2021 Published: 23 February 2022

Citation:

Zhang Q, Dong G, Meng W, Chen Z, Cao $Y$ and Zhang M (2022) Perceived

Stress and Psychological Impact

Among Healthcare Workers at a

Tertiaty Hospital in China During the COVID-19 Outbreak: The Moderating Role of Resilience and Social Support.

Front. Psychiatry 12:570971

doi: 10.3389/fpsyt.2021.570971
Aims: To investigate the psychological distress experienced by healthcare workers (HCWs) at a tertiary hospital in Changzhou, China, outside Wuhan, during the early stage of COVID-19 and evaluate the moderating effects of resilience and social support on the relationship between stress and psychological distress.

Methods: The study was conducted between February 10 and 15, 2020, in a non-probabilistic way. The survey included questions regarding the risk of exposure, sociodemographics, perceived stress [10-item Perceived Stress Scale (PSS10)], resilience [10-item Connor-Davidson Psychological Resilience (CD-RISC-10)], social support [Multidimensional Scale of Perceived Social Support (MSPSS)], and psychological distress [12-item General Health Questionnaire (GHQ-12)]. We applied the PROCESS macro for SPSS to test the hypotheses that resilience and social support moderated the stress response. In addition, a simple slope analysis was conducted when the interaction effect was statistically significant.

Results: Some $33.6 \%$ of participants suffered from psychological distress (GHQ-12 $\geq 12)$. Perceived stress was positively related to psychological distress $(r=0.42, p<$ $0.001)$. In addition, resilience $\left(\Delta R^{2}=0.03, p\right.$ for interaction $\left.<0.001\right)$ and social support $\left(\Delta \mathrm{R}^{2}=0.01, p\right.$ for interaction $\left.<0.01\right)$ moderated the stress response. The impact of perceived stress on psychological distress was attenuated when subjects who were resilient (high $\beta=0.15, p<0.001$; low $\beta=0.36, p<0.001$ ), and perceived stress had less impact on psychological distress when social support was high $(\beta=0.24, p<$ $0.001)$ rather than low $(\beta=0.34, p<0.001)$.

Limitations: The cross-sectional design led to a lack of causal relationships between variables.

Conclusions: Our data showed that resilience and social support moderated the stress response among HCWs in the pandemic, suggesting that improving resilience and social support could be appropriate targets to improve HCWs' mental health in the pandemic.

Keywords: 2019 novel coronavirus disease, healthcare workers, perceived stress, psychological distress, resilience, social support 


\section{INTRODUCTION}

Wuhan, China, was hit with cases of life-threatening pneumonia caused by the novel coronavirus (COVID-19) in December 2019. Researchers found that the COVID-19 virus was more contagious than SARS, so COVID-19 quickly swept into every province in China. According to the Chinese government report, it had infected 58,016 people on February 17, 2020, and had caused more than 3,000 deaths within 2 months $(1,2)$. In addition, since healthcare workers (HCWs) have been the main force of the battle against the coronavirus, at least 1,716 HCWs had been infected with the virus by February 14, 2020, including six deaths (3).

A pandemic causes severe physical problems and different degrees of psychological distress $(4,5)$. While our hospital is located outside Wuhan, 30 cases have been confirmed in our city. As one of the biggest tertiary hospitals, our HCWs experienced an increasing workload and stress, especially when the knowledge about the disease transmission, disease course, and pathogenesis was limited during the first outbreak. Therefore, it has clinical significance to assess their mental health $(5,6)$. We applied the 12-item General Health Questionnaire (GHQ-12) to assess the severity of psychological distress among the HCWs in this hospital.

It is well known that different people react in different ways to stress. An individual's biological vulnerability, resilience, and social support could affect the psychological impact (7). As an ability to "bounce back", resilience may strengthen stress resistance. Having a higher level of resilience leads to better mental health and decreased stress $(8,9)$. Individuals who receive social support typically get it from their family, friends, and other members of society. Social support can potentially improve coping with life stress and is highly associated with psychological status. We assumed that stress is related to psychological distress, and resilience and social support moderate their relationship.

In this study, we investigated the psychological distress among HCWs in our hospital when facing the first outbreak of COVID19. In addition, we examined the effects of the moderators in the stress response, hoping to find effective buffers to avoid the psychological impact induced by the COVID-19 outbreak.

\section{METHODS}

\section{Sample Size}

We applied the "Confidence Intervals for One Proportion" module of PASS software to calculate the sample size $(10,11)$. A sample size of 402 was calculated by a two-sided $95 \%$ confidence interval with a width equal to 0.1 when the sample proportion is $50 \%$. The sample proportion is the estimated prevalence of psychological distress in our hospital based on other studies (12).

\section{Study Design}

The questionnaire was uploaded to "Wenjuanxing" (www.wjx.cn), a secure survey website. To protect the privacy of the individuals, we kept the obtained data confidential and anonymous. The first page of the survey was an introduction of the study. Before conducting the survey, subjects were required to read and agree to the statement. Since the survey was openended, respondents were free to drop if they wished. All surveys with complete answers were allowed to be submitted. Depending on the Internet Protocol (IP) recorded by the online platform, a participant could submit only one time.

\section{Subjects and Procedure}

The sample was recruited from February 10-15, 2020, and selected in a non-probabilistic way. We distributed the online survey link to staff via our hospital's official WeChat account. Besides, to increase the response rate, we encouraged the leadership of all departments to forward online questionnaire to all HCWs by WeChat groups. Finally, we obtained 1,098 responses from our electronic questionnaire. After removing 34 incomplete questionnaires, 1,064 individuals remained in our analyses.

The Clinical Research Ethics Committee of the Affiliated Changzhou No.2 People's Hospital of Nanjing Medical University approved the study.

\section{Measurements}

Demographic information included age, gender, education, working occupation, chronic diseases, marital status, living with children, double staff, and major life events within half a year. Working occupations included doctors, nurses, technicians, and others (administrative and year-service staff). Regarding the risk of exposure, high risk was defined as working in the fever clinics, emergency department, suspected COVID-19 isolation units, and medical rescue team rushed to Wuhan.

Psychological distress was assessed by GHQ-12. The fourpoint Likert scoring method was applied in this study. Item responses range from 0 to 3 , and the overall score ranges from 0 to 36 , with more than 12 being defined as cases (12). The Chinese version has been validated in different groups of Chinese (13-15).

Perceived stress was measured by the 10-item Perceived Stress Scale (PSS-10). Participants are asked to report how they felt and thought during the last month. Each item ranges from 0 to 4, and overall score ranges from 0 to 40 (16). A large sample study showed that PSS-10 has good psychometric properties in Chinese populations (17).

Resilience was assessed by the 10-item Connor-Davidson Psychological Resilience (CD-RISC-10). Each item ranges from 0 to 4 . The Chinese version has been validated $(18,19)$.

Social support was evaluated by the Multidimensional Scale of Perceived Social Support (MSPSS), a 12-item questionnaire. Each item ranges from 0 to 6 . The Chinese version of the MSPSS has been shown to have excellent reliability and validity (20).

\section{Statistics}

We applied SPSS 23.0 to conduct the analyses. Mean \pm SD was used to describe the continuous variables. Categorical variables were expressed as frequencies (proportion). We used the independent-sample $t$-test and chi-square test to compare the non-case and case groups. Pearson's correlation analysis was applied in analyzing the relationship between key variables.

To test the hypotheses about moderating effect, we applied the PROCESS macro for SPSS, using the moderating Model 
TABLE 1 | Sociodemographic of the participants $(N=1,064)$.

\begin{tabular}{|c|c|c|c|c|c|}
\hline & \multirow[t]{2}{*}{ Total $(n=1,064)$} & \multicolumn{2}{|c|}{ GHQ-12 } & \multirow[t]{2}{*}{$t / \chi^{2}$} & \multirow[t]{2}{*}{$p$} \\
\hline & & Non-case $[n(\%)]$ & Case $[n(\%)]$ & & \\
\hline Risk of exposure, $n(\%)$ & & & & 17.198 & 0.000 \\
\hline High risk & 345 & $199(57.7)$ & $146(42.3)$ & & \\
\hline Low risk & 719 & $507(70.5)$ & $212(29.5)$ & & \\
\hline Age, years, mean $\pm S D$ & $33.3 \pm 8.2$ & $32.9 \pm 8.3$ & $34.2 \pm 8.1$ & -2.511 & 0.012 \\
\hline \multicolumn{6}{|l|}{ Gender, $n(\%)$} \\
\hline Male & 180 & $127(70.6)$ & $53(29.4)$ & 1.714 & 0.191 \\
\hline Female & 884 & $579(65.5)$ & 305 (34.5) & & \\
\hline Occupation, $n(\%)$ & & & & 9.725 & 0.021 \\
\hline Doctor & 295 & $183(62.0)$ & $112(38.0)$ & & \\
\hline Nurse & 685 & $459(67.0)$ & 226 (33.0) & & \\
\hline Technician & 10 & $5(50.0)$ & $5(50.0)$ & & \\
\hline Other & 74 & $59(79.7)$ & $15(20.3)$ & & \\
\hline Education, $n(\%)$ & & & & 12.371 & 0.002 \\
\hline High school or less & 17 & $6(35.3)$ & $11(64.7)$ & & \\
\hline Undergraduate university or college & 829 & $568(68.5)$ & $261(31.5)$ & & \\
\hline Medical or graduate school & 218 & $132(60.6)$ & $86(39.4)$ & & \\
\hline Chronic diseases, $n(\%)$ & & & & 11.992 & 0.001 \\
\hline Yes & 97 & $49(50.5)$ & $48(49.5)$ & & \\
\hline No & 967 & $657(67.9)$ & $310(32.1)$ & & \\
\hline Marital status, $n(\%)$ & & & & 10.470 & 0.015 \\
\hline Unmarried & 288 & $213(74.0)$ & $75(26.0)$ & & \\
\hline Married & 746 & $475(63.7)$ & 271 (36.3) & & \\
\hline Divorced & 27 & $16(59.3)$ & $11(40.7)$ & & \\
\hline Widowed & 3 & $2(66.7)$ & $1(33.3)$ & & \\
\hline Living with children, $n(\%)$ & & & & 15.105 & 0.000 \\
\hline Yes & 672 & $417(62.1)$ & 255 (37.9) & & \\
\hline No & 392 & $289(73.7)$ & 103 (26.3) & & \\
\hline Double staff, $n(\%)$ & & & & 2.463 & 0.117 \\
\hline Yes & 238 & 168 (70.6) & $70(29.4)$ & & \\
\hline No & 826 & $538(65.1)$ & 288 (34.9) & & \\
\hline Major life events, $n$ (\%) & & & & 16.147 & 0.000 \\
\hline Yes & 125 & $63(50.4)$ & $62(49.6)$ & & \\
\hline No & 939 & $643(68.5)$ & 296 (31.5) & & \\
\hline PSS-10, mean \pm SD & $16.39 \pm 5.65$ & $14.98 \pm 5.39$ & $19.18 \pm 5.08$ & -12.244 & 0.000 \\
\hline \multicolumn{6}{|l|}{ MSPSS, mean \pm SD } \\
\hline Family supporting & $22.35 \pm 4.70$ & $23.08 \pm 4.42$ & $20.89 \pm 4.91$ & 7.109 & 0.000 \\
\hline Friend supporting & $21.49 \pm 4.58$ & $22.31 \pm 4.30$ & $19.87 \pm 4.70$ & 8.491 & 0.000 \\
\hline Other supporting & $20.73 \pm 4.63$ & $21.65 \pm 4.40$ & $18.91 \pm 4.53$ & 9.506 & 0.000 \\
\hline CD-RISC-10, mean \pm SD & $27.31 \pm 6.98$ & $29.32 \pm 6.62$ & $23.34 \pm 5.91$ & 14.437 & 0.000 \\
\hline
\end{tabular}

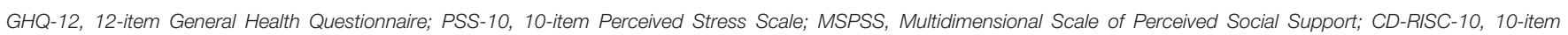
Connor-Davidson Resilience Scale.

$1(21,22)$, To determine if the moderation effect exists, the relationships for (i), (ii), and (iii) had to be significant-(i) direct effect of the predictor (perceived stress) on the outcome (psychological distress), (ii) direct effect of the moderator (social support or resilience) on psychological distress, and (iii) direct interactions effect (predictor $\times$ moderator) on psychological distress. PROCESS automatically calculates moderating effects and gives the change of $R^{2}\left(\Delta R^{2}\right)$ due to interaction. If statistically significant, the slope analysis was carried out. The sociodemographic correlates were entered as control variables in the moderation model. $p<0.05$ was statistically significant.

\section{RESULTS}

\section{Descriptive Analysis}

As shown in Table 1, among the 1,064 valid questionnaires, 358 (33.6\%) staff had symptoms of psychological distress. The rate of psychological distress was significantly higher in the high-risk 
TABLE 2 | Correlations between key variables

\begin{tabular}{llll}
\hline & $\mathbf{1}$ & $\mathbf{2}$ & $\mathbf{3}$ \\
\hline 1 Perceived stress & - & & \\
2 Resilience & $-0.21^{\star \star \star}$ & - & - \\
3 Social support & $-0.09^{\star \star}$ & $0.53^{\star \star \star}$ & $-0.28^{\star \star \star}$ \\
4 Psychological distress & $0.42^{\star \star \star}$ & $-0.48^{\star \star \star}$ & \\
${ }^{\star \star} p<0.01 ;{ }^{\star \star \star} p<0.001$. & & &
\end{tabular}

TABLE 3 | Moderating effect of resilience.

\begin{tabular}{|c|c|c|c|}
\hline Effect $^{a}$, variable & $R^{2}\left(\Delta R^{2}\right)$ & $F$ & $\beta$ \\
\hline $\begin{array}{l}\text { The direct effect of } \\
\text { perceived stress on } \\
\text { psychological distress }\end{array}$ & 0.07 & 12.27 & $0.31^{\star \star \star}$ \\
\hline $\begin{array}{l}\text { The direct effect of } \\
\text { resilience on psychological } \\
\text { distress }\end{array}$ & $0.35\left(0.28^{\star \star \star}\right)$ & 71.63 & $-0.40^{\star \star \star}$ \\
\hline $\begin{array}{l}\text { The direct interactive effect } \\
\text { (perceived stress } \times \\
\text { resilience) on psychological } \\
\text { distress }\end{array}$ & $0.38\left(0.03^{\star \star \star}\right)$ & 70.77 & $-0.16^{\star \star \star}$ \\
\hline
\end{tabular}

${ }^{\star \star \star} p<0.001$

${ }^{a}$ Adjusted for the sociodemographic.

exposure group than the low-risk group. Compared with the noncases, the cases had a higher level of perceived stress but less resilience or social support (all $p<0.05$ ). Besides, the proportion of chronic diseases, living with children, and major life events of cases were significantly higher than non-cases.

\section{Correlation Analysis Among Key Variables}

Table 2 shows intercorrelations among variables. Perceived stress was positively associated with psychological distress $(r=$ $0.42, p<0.001)$. Besides, psychological distress was negatively associated with resilience $(r=-0.48, p<0.001)$ and social support $(r=-0.28, p<0.001)$.

\section{Moderating Effect of Resilience}

As shown in Table 3, resilience $\left(\Delta \mathrm{R}^{2}=0.03, p\right.$ for interaction $<0.001)$ moderated in the relationship between perceived stress and psychological distress. Moreover, the impacts of perceived stress on psychological distress were attenuated when resilience was high (high $\beta=0.15, p<0.001$; low $\beta=0.36, p<0.001$ ). The interaction is visualized in Figure 1.

\section{Moderating Effect of Social Support}

Table 4 shows that social support moderated in the relationship between perceived stress and psychological distress $\left(\Delta \mathrm{R}^{2}=0.01\right.$, $p$ for interaction $<0.01)$. As shown in Figure 2, perceived stress had less impacts on psychological distress when social support was high $(\beta=0.24, p<0.001)$ rather than low $(\beta=0.34, p<$ $0.001)$.

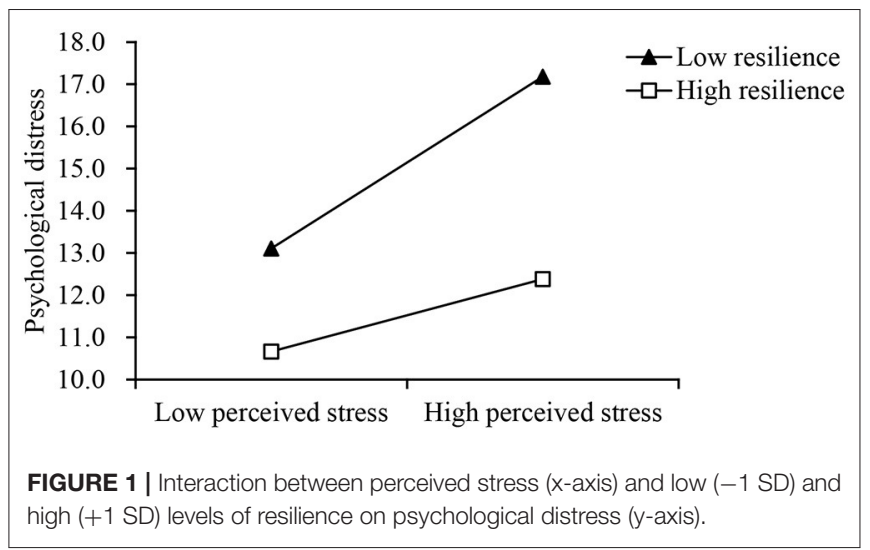

TABLE 4 | Moderating effect of social support.

\begin{tabular}{|c|c|c|c|}
\hline Effect ${ }^{a}$, variable & $R^{2}\left(\Delta R^{2}\right)$ & $\boldsymbol{F}$ & $\beta$ \\
\hline $\begin{array}{l}\text { The direct effect of } \\
\text { perceived stress on } \\
\text { psychological distress }\end{array}$ & 0.07 & 12.27 & $0.37^{\star \star \star}$ \\
\hline $\begin{array}{l}\text { The direct effect of social } \\
\text { support on psychological } \\
\text { distress }\end{array}$ & $0.27\left(0.19^{\star \star \star}\right)$ & 45.45 & $-0.25^{\star \star \star}$ \\
\hline $\begin{array}{l}\text { The direct interactive effect } \\
\text { (perceived stress } \times \text { social } \\
\text { support) on psychological } \\
\text { distress }\end{array}$ & $0.26\left(0.01^{\star \star}\right)$ & 41.84 & $-0.08^{\star \star}$ \\
\hline
\end{tabular}

${ }^{\star *} p<0.01 ;{ }^{* * *} p<0.001$.

a Adjusted for the sociodemographic.

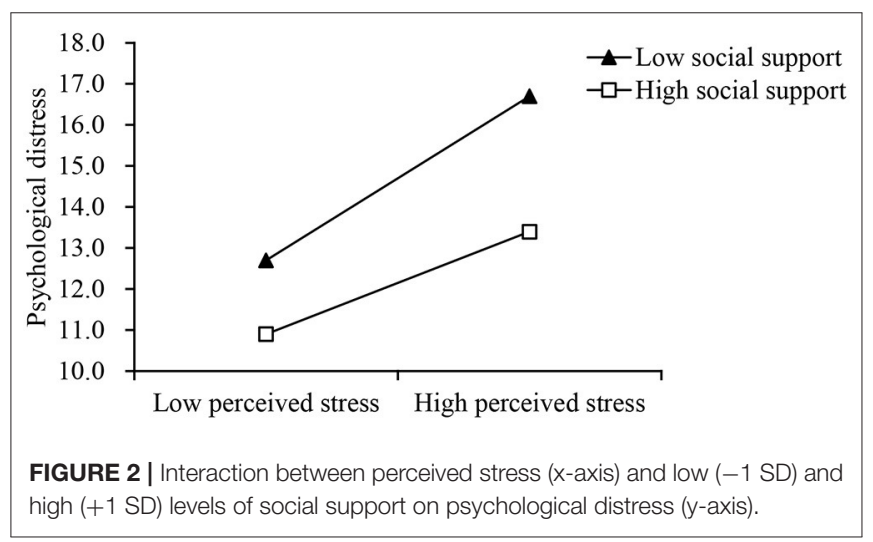

\section{DISCUSSION}

The present study found that $33.6 \%$ HCWs at this tertiary hospital experienced psychological distress during the first outbreak of COVID-19. In addition, the perceived stress was related to a higher psychological distress. Notably, resilience and social support moderated the stress response.

A mental health assessment of frontline HCWs is necessary to manage their stress and mental distress properly. A metaanalysis of studies conducted by April 17, 2020, found that the 
rate of depression and anxiety among HCWs in the pandemic was 23.2 and $22.8 \%$, respectively (23). Notably, Feng et al. applied the GHQ-12 to examine the mental health of staff from 26 hospitals in Shanghai between February 9 and 21, 2020, and found that the rate of psychological distress was $47.7 \%$ (12). Considering Changzhou is relatively near to Shanghai $(200 \mathrm{~km})$, and both studies were conducted during the early stages of the pandemic, the result of our study was similar to what was found in Shanghai.

The present study also identified two moderators: resilience and social support, in the stress response among the HCWs during the outbreak of the COVID-19. Besides, data showed that the stress response was weakened with high resilience or social support. These findings were similar to other studies. A study in the Philippines reported that nurses with higher resilience and social support showed lower COVID19 anxiety (24). Luceño-Moreno et al. found that resilience was the protective factor for Spanish HCWs' mental health (25). Notably, a large convenience sample study in the United States showed that HCWs who were more resilient had fewer COVID-19-related concerns, and higher resilience resulted in reduced anxiety and depression (26). Alnazly et al. reported significant correlations between social support and stress, anxiety, and depression among Jordanian HCWs in the pandemic (27).

During the early stage of the pandemic, HCWs may suffer from a decline in perceived support from friends or family due to the risk of infecting others and being isolated. Peer support appears to be necessary at this particular period, including support from colleagues and the hospital. A study in the Republic of Cyprus reported that organizational support was associated with HCWs' mental distress (28). In addition, another study in New York found that higher leadership support was linked to the lowest risk of depression, anxiety, and COVID-19-related post-traumatic symptoms (29). Therefore, the Columbia University Irving Medical Center program offers One-to-One Peer Support Sessions and Peer Support Groups to cope with psychological challenges in the pandemic (30). Besides, a hospital in France organized a safe place for HCWs to relax and support each other (31).

Meanwhile, building and maintaining resilience is essential for HCWs to recover quickly from the COVID19 pandemic (32). The University of Minnesota Medical Center proposed a psychological resilience intervention to attenuate psychological distress for HCWs in the pandemic, founded on a system (Battle Buddies) developed by the American Army (33). In addition, researchers reviewed resilience strategies to cope with stress and mental health in the pandemic (34). They suggested several appropriate approaches, including mindfulness, resilience training, and staff feedback sessions. Notably, since the researchers successfully tested the effect of computer-assisted resilience training, intervention programs that apply this tool are needed to help HCWs.

\section{LIMITATION}

This study has several potential limitations. The nonprobabilistic sampling we used may somewhat hinder the validity of our findings, as has been reported in many published literatures during the early stage of the pandemic $(26,35,36)$. However, our sample's prevalence of psychological distress was close to that of other studies conducted during the same period, indicating that our sample was somewhat representative of all HCWs in our hospital (12). In addition, the cross-sectional design led to a lack of causal relationships between variables. Moreover, residual confounding may exist because other factors could influence the outcome, such as income, coping skills, depression, anxiety, etc. Lastly, since this study was confined to a specific area (Changzhou), it will be necessary to conduct more studies to replicate these results.

\section{CONCLUSIONS}

Overall, this study found that resilience and social support could moderate stress response among HCWs during the pandemic. To decrease the stress response in the pandemic, the psychosocial intervention for HCWs should pay much attention to the improvement of resilience and social support.

\section{DATA AVAILABILITY STATEMENT}

The raw data supporting the conclusions of this article will be made available by the authors, without undue reservation.

\section{ETHICS STATEMENT}

The studies involving human participants were reviewed and approved by Institutional Review Board of Changzhou No.2 People's Hospital. The patients/participants provided their written informed consent to participate in this study.

\section{AUTHOR CONTRIBUTIONS}

MZ designed the study. QZ conducted the data analysis and wrote the manuscript. YC directed all the work. All authors contributed to the article and approved the submitted version.

\section{FUNDING}

This work was funded by Application Foundation Program of Changzhou Science and Technology (CJ20180071). 


\section{REFERENCES}

1. Zhao X, Lan M, Li H, Yang J. Perceived stress and sleep quality among the non-diseased general public in China during the 2019 coronavirus disease: a moderated mediation model. Sleep Med. (2021) 77:33945. doi: 10.1016/j.sleep.2020.05.021

2. Liu Y, Gayle AA, Wilder-Smith A, Rocklöv J. The reproductive number of COVID-19 is higher compared to SARS coronavirus. J Travel Med. (2020) 27:taaa021. doi: $10.1093 / \mathrm{jtm} / \mathrm{taaa} 021$

3. The epidemiological characteristics of an outbreak of 2019 novel coronavirus diseases (COVID-19) in China. Zhonghua liu xing bing xue za zhi. (2020) 41:145-51. doi: 10.3760/cma.j.issn.0254-6450.2020. 02.003

4. Bao Y, Sun Y, Meng S, Shi J, Lu L. 2019-nCoV epidemic: address mental health care to empower society. Lancet. (2020) 395:e37-8. doi: 10.1016/S0140-6736(20)30309-3

5. Chen Q, Liang M, Li Y, Guo J, Fei D, Wang L, et al. Mental health care for medical staff in China during the COVID-19 outbreak. lancet Psychiatry. (2020) 7:e15-6. doi: 10.1016/S2215-0366(20)30078-X

6. Du J, Dong L, Wang T, Yuan C, Fu R, Zhang L, et al. Psychological symptoms among frontline healthcare workers during COVID-19 outbreak in Wuhan. Gen Hosp Psychiatry. (2020) 67:144-5. doi: 10.1016/j.genhosppsych.2020. 03.011

7. Schneiderman N, Ironson G, Siegel SD. Stress and health: psychological, behavioral, and biological determinants. Annu Rev Clin Psychol. (2005) 1:607-28. doi: 10.1146/annurev.clinpsy.1.102803. 144141

8. Kermott CA, Johnson RE, Sood R, Jenkins SM, Sood A. Is higher resilience predictive of lower stress and better mental health among corporate executives? PLoS ONE. (2019) 14:e0218092. doi: 10.1371/journal.pone.0218092

9. Manchia M, Gathier AW, Yapici-Eser H, Schmidt MV, de Quervain D, van Amelsvoort $\mathrm{T}$, et al. The impact of the prolonged COVID-19 pandemic on stress resilience and mental health: a critical review across waves. Eur Neuropsychopharmacol. (2021) 55:22-83. doi: 10.1016/j.euroneuro.2021.10.864

10. Newcombe RG. Two-sided confidence intervals for the single proportion: comparison of seven methods. Stat Med. (1998) 17:85772. doi: 10.1002/(SICI) 1097-0258(19980430)17:8<857::AID-SIM777>3.0.CO;2E

11. Fleiss JL, Levin B, Paik MC. Statistical Methods for Rates and Proportions. 3rd ed. New York, NY: John Wiley \& Sons (2003).

12. Feng J, Xu J, Xu S, Cao H, Zheng C, Sharma L, et al. Psychological impact during the first outbreak of COVID-19 on frontline health care workers in Shanghai. Front Public Health. (2021) 9:646780. doi: 10.3389/fpubh.2021.646780

13. Liang $\mathrm{Y}$, Wang $\mathrm{L}$, Yin $\mathrm{X}$. The factor structure of the 12-item general health questionnaire (GHQ-12) in young Chinese civil servants. Health Qual Life Outcomes. (2016) 14:136. doi: 10.1186/s12955-016$0539-\mathrm{y}$

14. Ip WY, Martin CR. Psychometric properties of the 12-item General Health Questionnaire (GHQ-12) in Chinese women during pregnancy and in the postnatal period. Psychol Health Med. (2006) 11:60-9. doi: 10.1080/13548500500155750

15. Guan M, Han B. Factor structures of general health questionnaire-12 within the number of kins among the rural residents in China. Front Psychol. (2019) 10:1774. doi: 10.3389/fpsyg.2019.01774

16. Cohen S, Kamarck T, Mermelstein R. A global measure of perceived stress. $J$ Health Soc Behav. (1983) 24:385-96. doi: 10.2307/2136404

17. Huang F, Wang H, Wang Z, Zhang J, Du W, Su C, et al. Psychometric properties of the perceived stress scale in a community sample of Chinese. BMC Psychiatry. (2020) 20:130. doi: 10.1186/s12888-020-02 520-4

18. Wang L, Shi Z, Zhang Y, Zhang Z. Psychometric properties of the 10-item Connor-Davidson resilience scale in Chinese earthquake victims. Psychiatry Clin Neurosci. (2010) 64:499-504. doi: 10.1111/j.1440-1819.2010.02130.x

19. Cheng C, Dong D, He J, Zhong X, Yao S. Psychometric properties of the 10-item Connor-Davidson Resilience Scale (CD-RISC-10) in Chinese undergraduates and depressive patients. J Affect Disord. (2020) 261:21120. doi: $10.1016 /$ j.jad.2019.10.018

20. Xiang Y, Wang W, Guan F. The relationship between child maltreatment and dispositional envy and the mediating effect of self-esteem and social support in young adults. Front Psychol. (2018) 9:1054. doi: 10.3389/fpsyg.2018.01054

21. Hayes AF. Introduction to Mediation, Moderation, and Conditional Process Analysis: A Regression-Based Approach. New York, NY: Guilford Press (2013).

22. Ong HL, Vaingankar JA, Abdin E, Sambasivam R, Fauziana R, Tan ME, et al. Resilience and burden in caregivers of older adults: moderating and mediating effects of perceived social support. BMC Psychiatry. (2018) 18:27. doi: $10.1186 / \mathrm{s} 12888-018-1616-\mathrm{z}$

23. Pappa S, Ntella V, Giannakas T, Giannakoulis VG, Papoutsi E, Katsaounou P. Prevalence of depression, anxiety, and insomnia among healthcare workers during the COVID-19 pandemic: a systematic review and metaanalysis. Brain Behav Immun. (2020) 88:901-7. doi: 10.1016/j.bbi.2020. 05.026

24. Labrague LJ, De Los Santos JAA. COVID-19 anxiety among front-line nurses: predictive role of organisational support, personal resilience and social support. J Nurs Manag. (2020) 28:1653-61. doi: 10.1111/jonm. 13121

25. Luceño-Moreno L, Talavera-Velasco B, García-Albuerne Y, Martín-García J. Symptoms of posttraumatic stress, anxiety, depression, levels of resilience and burnout in spanish health personnel during the COVID-19 pandemic. Int $J$ Environ Res Public Health. (2020) 17:5514. doi: 10.3390/ijerph17155514

26. Barzilay R, Moore TM, Greenberg DM, DiDomenico GE, Brown LA, White LK, et al. Resilience, COVID-19-related stress, anxiety and depression during the pandemic in a large population enriched for healthcare providers. Transl Psychiatry. (2020) 10:291. doi: 10.1038/s41398-020-00982-4

27. Alnazly E, Khraisat OM, Al-Bashaireh AM, Bryant CL. Anxiety, depression, stress, fear and social support during COVID-19 pandemic among Jordanian healthcare workers. PLoS ONE. (2021) 16:e247679. doi: 10.1371/journal.pone.0247679

28. Chatzittofis A, Constantinidou A, Artemiadis A, Michailidou K, Karanikola MNK. The role of perceived organizational support in mental health of healthcare workers during the COVID-19 pandemic: a cross-sectional study. Front Psychiatry. (2021) 12:707293. doi: 10.3389/fpsyt.2021.707293

29. Feingold JH, Peccoralo L, Chan CC, Kaplan CA, Kaye-Kauderer H, Charney D, et al. Psychological impact of the COVID-19 pandemic on frontline health care workers during the pandemic surge in New York City. Chronic Stress. (2021) 5:2470547020977891. doi: 10.1177/2470547020977891

30. Mellins CA, Mayer LES, Glasofer DR, Devlin MJ, Albano AM, Nash SS, et al. Supporting the well-being of health care providers during the COVID-19 pandemic: the CopeColumbia response. Gen Hosp Psychiatry. (2020) 67:629. doi: 10.1016/j.genhosppsych.2020.08.013

31. Lefèvre H, Stheneur C, Cardin C, Fourcade L, Fourmaux C, Tordjman E, et al. The bulle: support and prevention of psychological decompensation of health care workers during the trauma of the COVID-19 epidemic. $J$ Pain Symptom Manage. (2021) 61:416-22. doi: 10.1016/j.jpainsymman.2020. 09.023

32. Rieckert A, Schuit E, Bleijenberg N, Ten Cate D, de Lange W, de Man-van Ginkel JM, et al. How can we build and maintain the resilience of our health care professionals during COVID-19? Recommendations based on a scoping review. BMJ Open. (2021) 11:e043718. doi: 10.1136/bmjopen-2020-043718

33. Albott CS, Wozniak JR, McGlinch BP, Wall MH, Gold BS, Vinogradov S. Battle buddies: rapid deployment of a psychological resilience intervention for health care workers during the COVID-19 pandemic. Anesth Analg. (2020) 131:43-54. doi: 10.1213/ANE.0000000000004912

34. Heath C, Sommerfield A, von Ungern-Sternberg BS. Resilience strategies to manage psychological distress among healthcare workers during the COVID-19 pandemic: a narrative review. Anaesthesia. (2020) 75:136471. doi: 10.1111/anae. 15180

35. Griffith GJ, Morris TT, Tudball MJ, Herbert A, Mancano G, Pike L, et al. Collider bias undermines our understanding of COVID-19 disease risk and severity. Nat Commun. (2020) 11:5749. doi: 10.1038/s41467-020-19478-2

36. Mediavilla R, Fernández-Jiménez E, Martínez-Alés G, Moreno-Küstner B, Martínez-Morata I, Jaramillo F, et al. Role of access to personal protective equipment, treatment prioritization decisions, and changes in job functions on health workers' mental health outcomes during the initial 
outbreak of the COVID-19 pandemic. J Affect Disord. (2021) 295:4059. doi: 10.1016/j.jad.2021.08.059

Conflict of Interest: The authors declare that the research was conducted in the absence of any commercial or financial relationships that could be construed as a potential conflict of interest.

Publisher's Note: All claims expressed in this article are solely those of the authors and do not necessarily represent those of their affiliated organizations, or those of the publisher, the editors and the reviewers. Any product that may be evaluated in this article, or claim that may be made by its manufacturer, is not guaranteed or endorsed by the publisher.

Copyright $\odot 2022$ Zhang, Dong, Meng, Chen, Cao and Zhang. This is an open-access article distributed under the terms of the Creative Commons Attribution License (CC $B Y)$. The use, distribution or reproduction in other forums is permitted, provided the original author(s) and the copyright owner(s) are credited and that the original publication in this journal is cited, in accordance with accepted academic practice. No use, distribution or reproduction is permitted which does not comply with these terms. 